Research Article

\title{
Nonlinear Vibration Analysis of Damaged Microplate considering Size Effect
}

\author{
Jihai Yuan, ${ }^{1,2}$ Xiangmin Zhang, ${ }^{2}$ and Changping Chen $\mathbb{D I D}^{1,2}$ \\ ${ }^{1}$ Department of Civil Engineering, Xiamen University, Xiamen, Fujian 361005, China \\ ${ }^{2}$ School of Civil Engineering and Architecture, Xiamen University of Technology, Xiamen, Fujian 361024, China \\ Correspondence should be addressed to Changping Chen; cpchen@hnu.edu.cn
}

Received 18 May 2020; Revised 1 August 2020; Accepted 11 August 2020; Published 26 August 2020

Academic Editor: Marco Alfano

Copyright (c) 2020 Jihai Yuan et al. This is an open access article distributed under the Creative Commons Attribution License, which permits unrestricted use, distribution, and reproduction in any medium, provided the original work is properly cited.

Since microplates are extensively used in MEMS devices such as microbumps, micromirrors, and microphones, this work aims to study nonlinear vibration of an electrically actuated microplate whose four edges are clamped. Based on the modified couple stress theory (MCST) and strain equivalent assumption, size effect and damage are taken into consideration in the present model. The dynamic governing partial differential equations of the microplate system were obtained using Hamilton's principle and solved using the harmonic balance method after they are transformed into ordinary differential equation with regard to time. Size effect and damage effect on nonlinear free vibration of the microplate under DC voltage are discussed using frequency-response curve. In the forced vibration analysis, the frequency-response curves were also employed for the purpose of highlighting the influence of different physical parameters such as external excitation, damping coefficient, material length scale parameter, and damage variable when the system is under AC voltage. The results presented in this study may be helpful and useful for the dynamic stability of a electrically actuated microplate system.

\section{Introduction}

Since a microelectromechanical system (MEMS) received a considerable amount of attention in recent years, numerous works related to nonlinear responses and characteristics because of intrinsic existence of nonlinearity of these microdevices have been carried out in recent years. Using the multiple scale method, Younis and Nayfeh [1] studied nonlinear vibration of a resonant microbeam under dynamic electrostatic force. Based on the same method, Abdel-Rahman and Nayfeh [2] studied response of a microresonant sensor actuated by superharmonic and subharmonic electric forces and their results provide an analytical solution to predict the resonant response. Zhang and Meng [3] proposed a simplified model in order to study the resonant responses and nonlinear dynamics of microcantilever under electronic excitation. An electromechanical coupled nonlinear dynamic response was presented by $\mathrm{Xu}$ and Jia [4], who employed the perturbation method to discuss influence of mechanical and electric parameters on nonlinear natural frequencies and vibrating amplitudes of a microbeam. Vogl and Nayfeh [5] reported the response of the clamped plate with circular shape under primary resonance excitation using a reduced-order model, which is general enough for effectively designing capacitive micromachined ultrasonic transducers. Nayfeh et al. [6] developed a novel model for a resonant gas sensor and studied its nonlinear dynamic characteristics. Jia et al. [7] conducted a parametric study on forced vibration of microswitches to show effects of Casimir force, residual stress, and geometrical nonlinearity on the frequency response characteristics. Kim et al. [8] examined resonant behaviors of a microbeam subjected to axial force and electrostatic force. Saghir and Younis [9] conducted a study on nonlinear vibration behavior of rectangular microplate under static and dynamic load. They found an interesting phenomenon where the microplate shows a hardening behavior which switches to softening as the DC load increases. Sheikhlou et al. [10] investigated nonlinear resonant behavior of diaphragm-type micropumps. 
However, size-dependent behavior of microstructures has been experimentally validated [11]. With this consideration, some interesting studies related to vibration behavior are presented. Using strain gradient theory and shear deformation theory, Arefi and Zenkour [12] presented free vibration of a piezoelectric laminated microbeam. Based on nonlocal piezoelasticity theory, Arefi and Zenkour presented size-dependent bending and vibration response of a sandwich piezomagnetic nanobeam with curvature [13] and without curvature [14]. They also investigated thermo-electro-magneto-mechanical bending behavior of a Sandwich nanoplate with simplysupported boundary conditions [15]. A nonclassical microplate model was proposed by Ke et al. [16], who employed MCST [17] to study nonlinear free vibration of annular microplate. Using the same theory, Ghayesh et al. [18] analyzed the nonlinear behavior of a size-dependent resonator under primary and superharmonic excitations. Using Eringen nonlocal theory, Arani and Jafari [19] analyzed nonlinear vibration of sandwich microplates resting on an elastic matrix. With application of different kinds of materials such as exponentially graded, piezoelectric, and magnetic, Sobhy and Zenkour [20], Arefi and Zenkour [21], and Arefi et al. [22] investigated size-dependent free vibration of sandwich microplate resting on different foundations. The nonlinear size-dependent static and dynamic behaviors of a MEMS device were presented by Farokhi and Ghayesh [23] on the basis of MCST. The free vibration characteristics of microplate with size dependency were displayed by Tahani et al. [24] based on the MCST recently with consideration of various effects such as couple stress components, electrostatic attraction, and different boundary conditions on both mode shapes and natural frequencies. Unlike the previous research, Veysi et al. [25] presented vibrational behavior of microdoubly curved shallow shells incorporated with von-Kármán geometric nonlinearity, size dependence, and shear deformation by adopting multiple scale method.

In the meantime, during manufacturing and operating process damage, that is, fatigue damage [26], discrete brittle damage [27] and contact damage [28] may occur due to the very existence of microcracks or abrasions which may evolve and develop [29]. However, most of these works ignored the significant role of size effect in mechanical behavior of microstructures. Actually, nonlinear size-dependent behavior of microstructure considering damage effect is limited in current literatures. Hence, a better understanding of damage and microscale effect on mechanical behavior of microstructure is necessary. With this motivation, this work aims to study size-dependent nonlinear free vibration and forced vibration behavior for microplates with damage under electrostatic actuation. In this research, the damage constitutive relations are established based on the strain equivalent assumption [30] and MCST. The governing differential equations were derived via Hamilton's principle and solved numerically by Galerkin's method and the harmonic balance method (HBM). The influence of various system parameters on nonlinear vibration of the microplate is studied comprehensively.

\section{Mathematical Modeling}

Consider a movable microplate with mass density $\rho$ over a stationary electrode under electronic force in Figure 1, where its width, length, and thickness are $b, a$, and $h$ separately. The initial gap and external voltage between the electrode and the microplate are $d$ and $V_{C}$ accordingly. The midsurface of the microplate is regarded as the reference plane $z=0$ as shown in this figure.

With the introduction of only one extra material parameter, material length scale parameter, Yang et al. [17] proposed MCST which claims the strain energy of an elastomer with volume $V$ is expressed as

$$
U=\frac{1}{2} \int_{V}\left(\sigma_{i j} \varepsilon_{i j}+m_{i j} \chi_{i j}\right) \mathrm{d} V, \quad(i, j=x, y, z),
$$

where stress tensor $\left(\sigma_{i j}\right)$, couple stress tensor $\left(m_{i j}\right)$, strain tensor $\left(\varepsilon_{i j}\right)$, and symmetric curvature tensor $\left(\chi_{i j}\right)$, are given as follows:

$$
\begin{aligned}
& \sigma_{i j}=\lambda \operatorname{tr}\left(\varepsilon_{i j}\right) \delta_{i j}+2 \mu \varepsilon_{i j}, \\
& m_{i j}=2 l^{2} \mu \chi_{i j}, \\
& \varepsilon_{i j}=\frac{1}{2}\left(u_{i, j}+u_{j, i}\right), \\
& \chi_{i j}=\frac{1}{2}\left(\theta_{i, j}+\theta_{j, i}\right),
\end{aligned}
$$

where $u_{i}$ represents displacement vector. There are two constitutive relations: one is between Cauchy's stress and infinitesimal engineering strain represented by (2) and the other is between couple stress and symmetric curvature represented (3). The former one is traditional elastic constitutive relation with lame constant $\lambda, \mu$. The latter one is extra constitutive relation accounting for size dependency using material length scale parameter $l$. The gyration vector $\left(\theta_{i}\right)$ is written as

$$
\theta_{i}=\frac{1}{2} e_{i j k} u_{k, j}
$$

where $e_{i j k}$ is the permutation tensor.

Kirchhoff's displacement components $u_{1}, u_{2}, u_{3}$ at a point of plate can be defined as

$$
\begin{aligned}
& u_{1}(x, y, z, t)=u(x, y, t)-z w_{x}, \\
& u_{2}(x, y, z, t)=v(x, y, t)-z w_{y}, \\
& u_{3}(x, y, z, t)=w(x, y, t),
\end{aligned}
$$

where $u, v$, and $w$ donate the displacements of the reference plane separately. For microplate encounters large deflection, the von Kármán's plate theory is adopted to describe the geometric nonlinearity of the microplate. Thus, the straindisplacement relations can be given as 


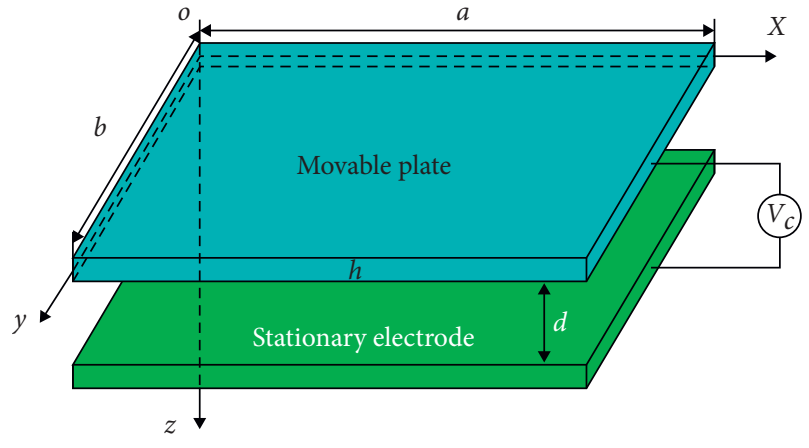

FIGURE 1: Electrically actuated microplate.

$$
\begin{aligned}
\varepsilon_{x} & =\varepsilon_{x}^{0}+z \kappa_{x}, \\
\varepsilon_{y} & =\varepsilon_{y}^{0}+z \kappa_{y}, \\
\varepsilon_{x y} & =\varepsilon_{x y}^{0}+z \kappa_{x y},
\end{aligned}
$$

where stains $\varepsilon_{x}^{0}, \varepsilon_{y}^{0}$, and $\varepsilon_{x y}^{0}$ and curvatures $\kappa_{x}, \kappa_{y}$, and $\kappa_{x y}$ at a point of the middle surface are expressed as

$$
\begin{aligned}
& \left\{\begin{array}{c}
\varepsilon_{x}^{0} \\
\varepsilon_{y}^{0} \\
\varepsilon_{x y}^{0}
\end{array}\right\}=\left\{\begin{array}{c}
u_{x}+\frac{1}{2} w_{x}^{2} \\
v_{y}+\frac{1}{2} w_{y}^{2} \\
u_{y}+v_{x}+w_{x} w_{y}
\end{array}\right\}, \\
& \left\{\begin{array}{c}
\kappa_{x} \\
\kappa_{y} \\
\kappa_{x y}
\end{array}\right\}=\left\{\begin{array}{c}
-w_{x x} \\
-w_{y y} \\
-2 w_{x y}
\end{array}\right\} .
\end{aligned}
$$

Substituting (7) into (6), one has gyration vector:

$$
\begin{aligned}
& \theta_{x}(x, y)=w_{y}, \\
& \theta_{y}(x, y)=-w_{x}, \\
& \theta_{z}(x, y)=\frac{1}{2}\left(v_{x}-u_{y}\right) .
\end{aligned}
$$

Substituting (10) into (2) yields curvature tensor:

$$
\left\{\begin{array}{l}
\chi_{x x} \\
\chi_{y y} \\
\chi_{x y} \\
\chi_{x z} \\
\chi_{y z}
\end{array}\right\}=\left\{\begin{array}{c}
w_{x y} \\
-w_{x y} \\
\frac{1}{2}\left(w_{y y}-w_{x x}\right) \\
\frac{1}{4}\left(v_{x x}-u_{x y}\right) \\
\frac{1}{4}\left(v_{x y}-u_{y y}\right)
\end{array}\right\} .
$$

The stress-strain relations can be given with respect to Poisson's ratio $v$ and Young's modulus $E$ as

$$
\begin{gathered}
\left\{\begin{array}{c}
\sigma_{x} \\
\sigma_{y} \\
\sigma_{x y}
\end{array}\right\}=Q^{d} \bullet\left\{\begin{array}{c}
\varepsilon_{x} \\
\varepsilon_{y} \\
\varepsilon_{x y}
\end{array}\right\}, \\
\left(m_{x x}, m_{y y}, m_{x y}, m_{x z}, m_{y z}\right)=F\left(\chi_{x x}, \chi_{y y}, \chi_{x y}, \chi_{x z}, \chi_{y z}\right),
\end{gathered}
$$

where

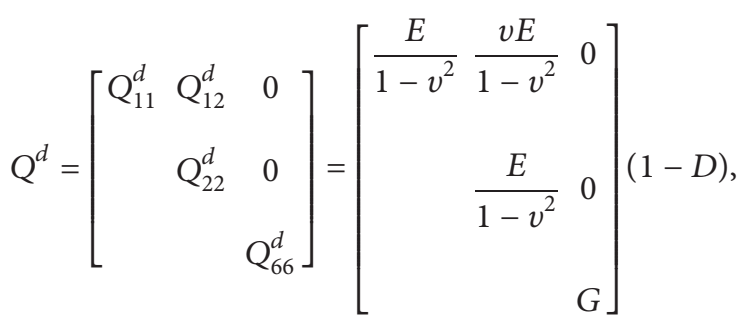

$$
F=2 l^{2} G(1-D)
$$

where $D$ denotes damage variable varying from 0 to 1 [30]; $G$ is shear modulus.

With Cauchy's stress and couple stress expressed above, bending moments $M_{x}, M_{y}, M_{x y}$, membrane stress resultants $N_{x}, N_{y}, N_{x y}$, and couple moments $Y_{x x}, Y_{y y}, Y_{x y}, Y_{x z}, Y_{y z}$ can be, respectively, defined as

$$
\begin{aligned}
& {\left[\begin{array}{lll}
M_{x} & M_{y} & M_{x y}
\end{array}\right]=\int_{-(h / 2)}^{h / 2} z\left[\begin{array}{lll}
\sigma_{x} & \sigma_{y} & \sigma_{x y}
\end{array}\right] \mathrm{d} z,} \\
& {\left[\begin{array}{lll}
N_{x} & N_{y} & N_{x y}
\end{array}\right]=\int_{-(h / 2)}^{h / 2}\left[\begin{array}{lll}
\sigma_{x} & \sigma_{y} & \sigma_{x y}
\end{array}\right] \mathrm{d} z,}
\end{aligned}
$$

$\left[\begin{array}{lllll}Y_{x x} & Y_{y y} & Y_{x y} & Y_{x z} & Y_{y z}\end{array}\right]=\int_{-(h / 2)}^{h / 2}\left[\begin{array}{lllll}m_{x x} & m_{y y} & m_{x y} & m_{x z} & m_{y z}\end{array}\right] \mathrm{d} z$.

Substituting (12) and (13) into (15) yields the following constitutive equations:

$$
\begin{aligned}
& \left\{\begin{array}{c}
N_{x} \\
N_{y} \\
N_{x y}
\end{array}\right\}=\left[\begin{array}{ccc}
A_{11} & A_{12} & 0 \\
A_{12} & A_{22} & 0 \\
0 & 0 & A_{66}
\end{array}\right]\left\{\begin{array}{c}
\varepsilon_{x}^{0} \\
\varepsilon_{y}^{0} \\
\varepsilon_{x y}^{0}
\end{array}\right\}, \\
& \left\{\begin{array}{c}
M_{x} \\
M_{y} \\
M_{x y}
\end{array}\right\}=\left[\begin{array}{ccc}
D_{11} & D_{12} & 0 \\
D_{12} & D_{22} & 0 \\
0 & 0 & D_{66}
\end{array}\right]\left\{\begin{array}{c}
\kappa_{x} \\
\kappa_{y} \\
\kappa_{x y}
\end{array}\right\}, \\
& {\left[\begin{array}{lllll}
Y_{x x} & Y_{y y} & Y_{x y} & Y_{x z} & Y_{y z}
\end{array}\right]=G\left[\begin{array}{lllll}
\chi_{x x} & \chi_{y y} & \chi_{x y} & \chi_{x z} & \chi_{y z}
\end{array}\right] \text {, }}
\end{aligned}
$$

where 


$$
\begin{aligned}
A_{i j} & =h Q_{i j}^{d}, \\
D_{i j} & =\left(\frac{h^{3}}{12}\right) Q_{i j}^{d}, \\
G^{\prime} & =h F, \\
(i, j & =1,2,6) .
\end{aligned}
$$

With Hamilton's principle, we have

$$
\delta \int_{t_{1}}^{t_{2}}(T-U+W) \mathrm{d} t=0 .
$$

Substituting stresses and strains into (1), the virtual strain energy is obtained as

$$
\delta U=\int_{V}\left(\sigma_{i j} \delta \varepsilon_{i j}\right) \mathrm{d} V+\int_{V}\left(m_{i j} \delta \chi_{i j}\right) \mathrm{d} V .
$$

The virtual kinetic energy is given as

$$
\delta T=\frac{1}{2} \rho \int_{V} \delta\left(w_{t}\right)^{2} \mathrm{~d} V .
$$

The virtual work done by electric force and damping force can be expressed as

$$
\delta W=\iint_{S}\left(F_{z}-c w_{t}\right) \delta w \mathrm{~d} S
$$

where $c$ denotes damping coefficient and $F_{z}$ represents the electric force defined as

$$
F_{z}=\frac{\varepsilon_{v} V_{C}^{2}}{2(d-w)^{2}},
$$

where $\varepsilon_{v}$ is the dielectric constant of air.

By substituting (19)-(21) into (18), one has the nonlinear dynamic equations of the microplate system as

$$
\begin{aligned}
& N_{x, x}+N_{x y, y}+\frac{1}{2} Y_{x z, x y}+\frac{1}{2} Y_{y z, y y}=0 \\
& N_{y, y}+N_{x y, x}-\frac{1}{2} Y_{x z, x x}-\frac{1}{2} Y_{y z, x y}=0 \\
& -\left(N_{x} w_{x}+N_{x y} w_{y}\right)_{x}-\left(N_{y} w_{y}+N_{x y} w_{x}\right)_{y}+\left(-M_{x}-Y_{x y}\right)_{x x} \\
& \quad+\left(-M_{y}+Y_{x y}\right)_{y y} \\
& +\left(-2 M_{x y}+Y_{x x}-Y_{y y}\right)_{x y}+F_{e}=\rho h w_{t t}+c w_{t} .
\end{aligned}
$$

Boundary conditions are as follows:

$$
\begin{aligned}
& N_{x}+\frac{1}{2} Y_{x z, y}=0 \\
& \text { or } \delta u=0 \text {, } \\
& N_{x y}-\frac{1}{2} Y_{x z, x}-\frac{1}{2} Y_{y z, y}=0, \\
& \text { or } \delta v=0 \text {, } \\
& \frac{1}{2} Y_{x z}=0 \text {, } \\
& \text { or } \delta v_{x}=0 \text {, } \\
& M_{x, x}+2 M_{x y, y}+N_{x} w_{x}+N_{x y} w_{y}+Y_{x y, x}+\left(-Y_{x x}+Y_{y y}\right)_{y}=0, \\
& \text { or } \delta w=0 \text {, } \\
& M_{x}+Y_{x y}=0 \text {, } \\
& \text { or } \delta w_{x}=0 \text {, } \\
& y=0, b \\
& N_{y}-\frac{1}{2} Y_{y z, x}=0, \\
& \text { or } \delta v=0 \\
& N_{x y}+\frac{1}{2} Y_{y z, y}+\frac{1}{2} Y_{x z, x}=0, \\
& \text { or } \delta u=0 \text {, } \\
& \frac{1}{2} Y_{y z}=0 \\
& \text { or } \delta u_{y}=0 \text {, } \\
& M_{y, y}+N_{y} w_{y}+N_{x y} w_{x}+2 M_{x y, x}-Y_{x y, y}-\left(Y_{x x}-Y_{y y}\right)_{x}=0 \\
& \text { or } \delta w=0 \text {, } \\
& M_{y}-Y_{x y}=0, \\
& \text { or } \delta w_{y}=0 \text {. }
\end{aligned}
$$

The following dimensionless parameters are introduced: 


$$
\begin{aligned}
& \xi=\frac{x}{a}, \\
& \eta=\frac{y}{b}, \\
& U=\frac{u}{a}, \\
& V=\frac{v}{b}, \\
& W=\frac{w}{h}, \\
& \lambda_{1}=\frac{h}{a}, \\
& \lambda_{2}=\frac{h}{b}, \\
& \bar{A}_{i j}=\frac{A_{i j}}{Q_{11} h}, \\
& \bar{l}=\frac{l}{h}, \\
& \bar{D}_{i j}=\frac{12 D_{i j}}{Q_{11} h^{3}}, \\
& \bar{G}_{1}=\frac{G^{\prime}}{8 G a^{3}}, \\
& \bar{G}_{2}=\frac{G^{\prime}}{8 G b^{3}}, \\
& \bar{G}_{3}=\frac{G^{\prime}}{2 G h^{3}}, \\
& \beta=\frac{G}{Q_{11}}, \\
& Q_{11}=\frac{E}{1-v^{2}}, \\
& \tau=\frac{t}{T}, \\
& \bar{C}=\frac{c a^{2} b^{2}}{Q_{11} h^{3} T}, \\
& \bar{V}_{C}=\frac{V_{C}}{V_{0}}, \\
& \alpha_{1}=\frac{\varepsilon_{v} a^{2} b^{2} V_{0}^{2}}{2 d^{2} h^{4} Q_{11}}, \\
& \alpha_{2}=\frac{\varepsilon_{v} a^{2} b^{2} V_{0}^{2}}{d^{3} h^{3} Q_{11}}, \\
& T=\frac{a b}{h} \sqrt{\frac{\rho}{Q_{11}}}, \\
& \omega=\Omega T,
\end{aligned}
$$


where $V_{0}$ is the unit voltage.

With application of Taylor series expansion into (22) and ignoring nonlinear terms with respect to $w$ [31], the non- dimensional governing equations of the microplate are written as

$$
\begin{aligned}
& \bar{A}_{11}(\left.U_{\xi \xi}+\lambda_{1}^{2} W_{\xi} W_{\xi \xi}\right)+\bar{A}_{12}\left(V_{\xi \eta}+\lambda_{2}^{2} W_{\eta} W_{\xi \eta}\right)+\beta \bar{A}_{66}\left(\frac{\lambda_{2}^{2}}{\lambda_{1}^{2}} U_{\eta \eta}+V_{\xi \eta}+\lambda_{2}^{2} W_{\xi} W_{\eta \eta}+\lambda_{2}^{2} W_{\eta} W_{\xi \eta}\right) \\
&+\frac{\beta}{\lambda_{1}} \bar{G}_{1}\left(V_{\xi \xi \xi \eta}-\frac{\lambda_{2}^{2}}{\lambda_{1}^{2}} U_{\xi \xi \eta \eta}+\frac{\lambda_{2}^{2}}{\lambda_{1}^{2}} V_{\xi \eta \eta \eta}-\frac{\lambda_{2}^{4}}{\lambda_{1}^{4}} U_{\eta \eta \eta \eta}\right)=0 \\
& \bar{A}_{12}(\left.U_{\xi \eta}+\lambda_{1}^{2} W_{\xi} W_{\xi \eta}\right)+\bar{A}_{22}\left(V_{\eta \eta}+\lambda_{2}^{2} W_{\eta} W_{\eta \eta}\right)+\beta \bar{A}_{66}\left(U_{\xi \eta}+\frac{\lambda_{1}^{2}}{\lambda_{2}^{2}} V_{\xi \xi}+\lambda_{1}^{2} W_{\eta} W_{\xi \xi}+\lambda_{1}^{2} W_{\xi} W_{\xi \eta}\right) \\
&-\frac{\beta}{\lambda_{2}} \bar{G}_{2}\left(\frac{\lambda_{1}^{4}}{\lambda_{2}^{4}} V_{\xi \xi \xi \xi}-\frac{\lambda_{1}^{2}}{\lambda_{2}^{2}} U_{\xi \xi \xi \eta}+\frac{\lambda_{1}^{2}}{\lambda_{2}^{2}} V_{\xi \xi \eta \eta}-U_{\xi \eta \eta \eta}\right)=0 \\
&\left(\frac{1}{12} \bar{D}_{11}+\beta \bar{G}_{3}\right) \frac{\lambda_{1}^{2}}{\lambda_{2}^{2}} W_{\xi \xi \xi \xi}+\left(\frac{1}{6} \bar{D}_{12}+\frac{1}{3} \bar{D}_{66}+2 \beta \bar{G}_{3}\right) W_{\xi \xi \eta \eta}+\left(\frac{1}{12} \bar{D}_{22}+\beta \bar{G}_{3}\right) \frac{\lambda_{2}^{2}}{\lambda_{1}^{2}} W_{\eta \eta \eta \eta}+W_{\tau \tau}+\bar{C} W_{\tau} \\
&=\left[\bar{A}_{11}\left(U_{\xi}+\frac{1}{2} \lambda_{1}^{2} W_{\xi}^{2}\right)+\bar{A}_{12}\left(V_{\eta}+\frac{1}{2} \lambda_{2}^{2} W_{\eta}^{2}\right)\right] \frac{1}{\lambda_{2}^{2}} W_{\xi \xi}+\left[\bar{A}_{11}\left(U_{\xi \xi}+\lambda_{1}^{2} W_{\xi} W_{\xi \xi}\right)+\bar{A}_{12}\left(V_{\xi \eta}+\lambda_{2}^{2} W_{\eta} W_{\xi \eta}\right)\right] \frac{1}{\lambda_{2}^{2}} W_{\xi} \\
&+2 \beta \bar{A}_{66}\left(\frac{1}{\lambda_{1}^{2}} U_{\eta}+\frac{1}{\lambda_{2}^{2}} V_{\xi}+W_{\eta} W_{\xi}\right) W_{\xi \eta}+\beta \bar{A}_{66}\left(\frac{1}{\lambda_{2}^{2}} V_{\xi \xi}+\frac{1}{\lambda_{1}^{2}} U_{\xi \eta}+W_{\eta} W_{\xi \xi}+W_{\xi} W_{\xi \eta}\right) W_{\eta} \\
&+\left[\bar{A}_{12}\left(U_{\xi}+\frac{1}{2} \lambda_{1}^{2} W_{\xi}^{2}\right)+\bar{A}_{22}\left(V_{\eta}+\frac{1}{2} \lambda_{2}^{2} W_{\eta}^{2}\right)\right] \frac{1}{\lambda_{1}^{2}} W_{\eta \eta}+\left[\bar{A}_{12}\left(U_{\xi \eta}+\lambda_{1}^{2} W_{\xi} W_{\xi \eta}\right)+\bar{A}_{22}\left(V_{\eta \eta}+\lambda_{2}^{2} W_{\eta} W_{\eta \eta}\right)\right] \frac{1}{\lambda_{1}^{2}} W_{\eta} \\
&+\beta \bar{A}_{66}\left(\frac{1}{\lambda_{1}^{2}} U_{\eta \eta}+\frac{1}{\lambda_{2}^{2}} V_{\xi \eta}+W_{\xi \eta} W_{\eta}+W_{\xi} W_{\eta \eta}\right) W_{\xi}+\left(\alpha_{1}+\alpha_{2} W\right) \bar{V}_{C}^{2} .
\end{aligned}
$$

Due to the fact that a fully clamped rectangular microplate is extensively used in MEMS applications [32],

this kind of boundary condition is also studied in this work and written in dimensionless formulation as follows:

$$
\begin{gathered}
\bar{A}_{11}\left(U_{, \xi}+\frac{1}{2} W_{, \xi}^{2}\right)+\frac{\beta \bar{G}_{3}}{8}\left(\frac{h^{2}}{a b} V_{, \xi \xi \eta}-\frac{h^{2}}{b^{2}} U_{, \xi \eta \eta}\right)=0, \quad \xi=0,1, V=0, W=0, V_{, \xi}=0, W_{, \xi}=0, \\
\bar{A}_{22}\left(V_{, \eta}+\frac{1}{2} \lambda_{1} W_{, \eta}^{2}\right)-\frac{\beta \bar{G}_{3}}{8}\left(\frac{h^{2}}{a^{2}} V_{, \xi \xi \eta}-\frac{h^{2}}{a b} U_{, \xi \eta \eta}\right)=0, \quad \eta=0,1, U=0, W=0, U_{, \eta}=0, W_{, \eta}=0 .
\end{gathered}
$$

\section{Solution Methodology}

First, note that, in the boundary conditions in (27), the items related to size effect are neglected due to the fact that they are negligible compared with other items [29]. With satisfaction of boundary conditions, a solution for (26) takes the following form:

$$
\begin{aligned}
U(\xi, \eta, \tau) & =\bar{U}(\tau) \cos \pi \xi \sin ^{2} \pi \eta, \\
V(\xi, \eta, \tau) & =\bar{V}(\tau) \sin ^{2} \pi \xi \cos \pi \eta \\
W(\xi, \eta, \tau) & =\bar{W}(\tau) \sin ^{2} \pi \xi \sin ^{2} \pi \eta
\end{aligned}
$$

Submitting (28) into (26)fd25 and multiplying them by $\cos \pi \xi \sin ^{2} \pi \eta, \sin ^{2} \pi \xi \cos \pi \eta, \quad$ and $\sin ^{2} \pi \xi \sin ^{2} \pi \eta$, 
respectively, and integrating them from 0 to 1 with respect to $\xi$ and $\eta$, the nonlinear governing differential equations with respect to time can be obtained as

$$
\begin{aligned}
& {\left[-\frac{3 \pi^{2}}{16} \bar{A}_{11}-\beta \bar{A}_{66} \frac{\pi^{2} \lambda_{2}^{2}}{4 \lambda_{1}^{2}}-\frac{\bar{G}_{1} \beta}{\lambda_{1}} \frac{\pi^{4} \lambda_{2}^{2}}{4 \lambda_{1}^{2}}+\frac{\pi^{4} \lambda_{2}^{4}}{\lambda_{1}^{4}}\right] \bar{U}+\left[-\frac{16}{9} \bar{A}_{12}-\frac{16}{9} \beta \bar{A}_{66}+\frac{\bar{G}_{1} \beta}{\lambda_{1}} \frac{64 \pi^{2}}{9}+\frac{16 \pi^{2} \lambda_{2}^{2}}{9 \lambda_{1}^{2}}\right] \bar{V}} \\
& +\left[\frac{\pi^{2}}{6} \lambda_{1}^{2} \bar{A}_{11}+\frac{2 \pi^{2}}{15} \lambda_{2}^{2} \bar{A}_{12}-\frac{2 \pi^{2}}{15} \beta \bar{A}_{66} \lambda_{2}^{2}\right] \bar{W}^{2}=0, \\
& {\left[-\frac{16}{9} \bar{A}_{12}-\frac{16}{9} \beta \bar{A}_{66}+\frac{\bar{G}_{2} \beta}{\lambda_{2}} \frac{16 \pi^{2} \lambda_{1}^{2}}{9 \lambda_{2}^{2}}+\frac{64 \pi^{2}}{9}\right] \bar{U}+\left[-\frac{3 \pi^{2}}{16} \bar{A}_{22}-\frac{\pi^{2} \lambda_{1}^{2}}{4 \lambda_{2}^{2}} \beta \bar{A}_{66}-\frac{\bar{G}_{2} \beta}{\lambda_{2}} \frac{\pi^{4} \lambda_{1}^{4}}{\lambda_{2}^{4}}+\frac{\pi^{4} \lambda_{1}^{2}}{4 \lambda_{2}^{2}}\right] \bar{V}} \\
& +\left[\frac{2 \pi^{2}}{15} \lambda_{1}^{2} \bar{A}_{12}+\frac{\pi^{2}}{6} \lambda_{2}^{2} \bar{A}_{22}-\frac{2 \pi^{2}}{15} \beta \bar{A}_{66} \lambda_{1}^{2}\right] \bar{W}^{2}=0, \\
& \left\{\left[3 \frac{1}{12} \bar{D}_{11}+\beta \bar{G}_{3} \frac{\lambda_{1}^{2}}{\lambda_{2}^{2}}+\frac{1}{6} \bar{D}_{12}+\frac{1}{3} \beta \bar{D}_{66}+2 \beta \bar{G}_{3}+3 \frac{1}{12} \bar{D}_{22}+\beta \bar{G}_{3} \frac{\lambda_{1}^{2}}{\lambda_{2}^{2}}\right] \frac{\pi^{4}}{4}\right\} \bar{W}+\frac{1}{4} \bar{W}_{\tau \tau}+\frac{\bar{C}}{4} \bar{W}_{\tau} \\
& =\frac{\pi^{2} \bar{A}_{11}}{3 \lambda_{2}^{2}}-\frac{4 \pi^{2} \beta \bar{A}_{66}}{15 \lambda_{1}^{2}}+\frac{4 \pi^{2} \bar{A}_{12}}{15 \lambda_{1}^{2}} \bar{U} \bar{W}+\frac{4 \pi^{2} \bar{A}_{12}}{15 \lambda_{2}^{2}}-\frac{4 \pi^{2} \beta \bar{A}_{66}}{15 \lambda_{2}^{2}}+\frac{\pi^{2} \bar{A}_{22}}{3 \lambda_{1}^{2}} \bar{V} \bar{W}_{-}-\frac{52.5 \pi^{4} \lambda_{1}^{2}}{1024 \lambda_{2}^{2}} \bar{A}_{11}+\frac{25 \pi^{4} \bar{A}_{12}}{1024} \\
& +\frac{52.5 \pi^{4} \lambda_{2}^{2}}{1024 \lambda_{1}^{2}} \bar{A}_{22}+\frac{50 \pi^{4}}{1024} \beta \bar{A}_{66} \bar{W}^{3}+\frac{1}{4} \alpha_{1}+\frac{9}{16} \alpha_{2} \bar{W}_{\bar{V}}^{2} \text {. }
\end{aligned}
$$

For convenience, (29) can be expressed as follows:

$$
\begin{aligned}
& M_{11} \bar{U}+M_{12} \bar{V}+M_{13} \bar{W}^{2}=0 \\
& M_{21} \bar{U}+M_{22} \bar{V}+M_{23} \bar{W}^{2}=0 \\
& M_{31} \bar{W}+\frac{1}{4} \bar{W}_{\tau \tau}+\frac{\bar{C}}{4} \bar{W}_{\tau}=M_{32} \bar{U} \bar{W}+M_{33} \bar{V} \bar{W}-M_{34} \bar{W}^{3}+\frac{1}{4} \alpha_{1}+\frac{9}{16} \alpha_{2} \bar{W} \bar{V}_{C}^{2},
\end{aligned}
$$

where

$$
\begin{aligned}
& M_{11}=-\frac{3 \pi^{2}}{16} \bar{A}_{11}-\beta \bar{A}_{66} \frac{\pi^{2} \lambda_{2}^{2}}{4 \lambda_{1}^{2}}-\frac{\bar{G}_{1} \beta}{\lambda_{1}}\left(\frac{\pi^{4} \lambda_{2}^{2}}{4 \lambda_{1}^{2}}+\frac{\pi^{4} \lambda_{2}^{4}}{\lambda_{1}^{4}}\right), \\
& M_{12}=-\frac{16}{9} \bar{A}_{12}-\frac{16}{9} \beta \bar{A}_{66}+\frac{\bar{G}_{1} \beta}{\lambda_{1}}\left(\frac{64 \pi^{2}}{9}+\frac{16 \pi^{2} \lambda_{2}^{2}}{9 \lambda_{1}^{2}}\right), \\
& M_{13}=\frac{\pi^{2}}{6} \lambda_{1}^{2} \bar{A}_{11}+\frac{2 \pi^{2}}{15} \lambda_{2}^{2} \bar{A}_{12}-\frac{2 \pi^{2}}{15} \beta \bar{A}_{66} \lambda_{2}^{2}, \\
& M_{21}=-\frac{16}{9} \bar{A}_{12}-\frac{16}{9} \beta \bar{A}_{66}+\frac{\bar{G}_{2} \beta}{\lambda_{2}}\left(\frac{16 \pi^{2} \lambda_{1}^{2}}{9 \lambda_{2}^{2}}+\frac{64 \pi^{2}}{9}\right),
\end{aligned}
$$




$$
\begin{aligned}
& M_{22}=-\frac{3 \pi^{2}}{16} \bar{A}_{22}-\frac{\pi^{2} \lambda_{1}^{2}}{4 \lambda_{2}^{2}} \beta \bar{A}_{66}-\frac{\bar{G}_{2} \beta}{\lambda_{2}}\left(\frac{\pi^{4} \lambda_{1}^{4}}{\lambda_{2}^{4}}+\frac{\pi^{4} \lambda_{1}^{2}}{4 \lambda_{2}^{2}}\right), \\
& M_{23}=\frac{2 \pi^{2}}{15} \lambda_{1}^{2} \bar{A}_{12}+\frac{\pi^{2}}{6} \lambda_{2}^{2} \bar{A}_{22}-\frac{2 \pi^{2}}{15} \beta \bar{A}_{66}, \\
& M_{31}=\left[3\left(\frac{1}{12} \bar{D}_{11}+\beta \bar{G}_{3}\right) \frac{\lambda_{1}^{2}}{\lambda_{2}^{2}}+\left(\frac{1}{6} \bar{D}_{12}+\frac{1}{3} \bar{D}_{66}+2 \beta \bar{G}_{3}\right)+3\left(\frac{1}{12} \bar{D}_{22}+\beta \bar{G}_{3}\right) \frac{\lambda_{1}^{2}}{\lambda_{2}^{2}}\right] \frac{\pi^{4}}{4}, \\
& M_{32}=\frac{\pi^{2} \bar{A}_{11}}{3 \lambda_{2}^{2}}-\frac{4 \pi^{2} \beta \bar{A}_{66}}{15 \lambda_{1}^{2}}+\frac{4 \pi^{2} \bar{A}_{12}}{15 \lambda_{1}^{2}}, \\
& M_{33}=\frac{4 \pi^{2} \bar{A}_{12}}{15 \lambda_{2}^{2}}-\frac{4 \pi^{2} \beta \bar{A}_{66}}{15 \lambda_{2}^{2}}+\frac{\pi^{2} \bar{A}_{22}}{3 \lambda_{1}^{2}}, \\
& M_{34}=-\frac{52.5 \pi^{4} \lambda_{1}^{2}}{1024 \lambda_{2}^{2}} \bar{A}_{11}-\frac{25 \pi^{4}}{1024} \bar{A}_{12}-\frac{52.5 \pi^{4} \lambda_{2}^{2}}{1024 \lambda_{1}^{2}} \bar{A}_{22}-\frac{50 \pi^{4}}{1024} \beta \bar{A}_{66} .
\end{aligned}
$$

After eliminating $\bar{U}(\tau), \bar{V}(\tau)$, the nonlinear differential where equation only related to $\bar{W}(\tau)$ can be obtained:

$$
\frac{1}{4} \bar{W}_{\tau \tau}+\frac{\bar{C}_{\bar{W}}}{4} \bar{W}_{\tau}+M_{31} \bar{W}-\varphi \bar{W}^{3}=\frac{1}{4}\left(\alpha_{1}+\frac{9}{16} \alpha_{2} \bar{W}\right) \bar{V}_{C}^{2},
$$

$$
\varphi=M_{34}+\frac{M_{32} M_{22} M_{13}-M_{32} M_{12} M_{23}}{M_{12} M_{21}-M_{11} M_{22}}+\frac{M_{33} M_{11} M_{23}-M_{33} M_{13} M_{21}}{M_{12} M_{21}-M_{11} M_{22}} .
$$

\section{Results and Discussion}

In this section, the nonlinear size-dependent vibration analysis of the microplate is studied under the influence of size effect and damage effect. Free vibration and forced vibration analysis of the microplate under $\mathrm{DC}$ voltage and $\mathrm{AC}$ voltage are discussed in detail accordingly. The frequency-response curves are presented for various system parameters such as material length scale parameter, damage variable, damping ratio, and external AC voltage. Note that geometric and physical parameters of the microplate are listed in Table 1.

4.1. Free Vibration Analysis under DC Voltage. For static analysis, (32) can be written as

$$
\frac{\bar{W}_{\tau \tau}+\bar{C} \bar{W}_{\tau}}{4}+M_{31} \bar{W}-\varphi \bar{W}^{3}=\frac{\left(\alpha_{1}+(9 / 16) \alpha_{2} \bar{W}\right) \bar{V}_{d}^{2}}{4},
$$

where $V_{D}$ donates the DC voltage.
The harmonic balance method is feasible for finding solutions of both weakly and strongly nonlinear problems. A general solution of (34) can be given as

$$
W(\tau)=A_{0}+A_{1} \cos (\omega \tau+\theta) .
$$

Submitting (35) into (34) and using harmonic balance method (HB) [33], we obtain

$$
\begin{aligned}
& \omega_{0}^{2} A_{0}-4 \varphi\left(A_{0}^{3}+\frac{3}{2} A_{0} A_{1}^{2}\right)=\alpha_{1} \bar{V}_{d}^{2} \\
& -A_{1} \omega^{2}+\omega_{0}^{2} A_{1}-4 \varphi\left(3 A_{0}^{2} A_{1}+\frac{3}{4} A_{1}^{3}\right)=0,
\end{aligned}
$$

where $A_{0}$ is so smaller than $A_{1}$ that it can be neglected in the further step of solution, and then the following is obtained:

$$
\begin{aligned}
& \omega_{0}^{2}=4 M_{31}-\frac{9}{16} \alpha_{2} \bar{V}_{d}^{2}, \\
& \omega^{2}=\omega_{0}^{2}-3 \varphi A_{1}^{2} .
\end{aligned}
$$


TABLE 1: Geometric and material properties of the microplate.

\begin{tabular}{lc}
\hline Property & Value \\
\hline Length, $a$ & $300 \mu \mathrm{m}$ \\
Width, $b$ & $150 \mu \mathrm{m}$ \\
Thickness, $h$ & $2 \mu \mathrm{m}$ \\
Initial gap, $d$ & $2 \mu \mathrm{m}$ \\
Poisson's ratio, $v$ & 0.06 \\
Young's modulus, $E$ & $166 \times 10^{9} \mathrm{~Pa}$ \\
$\beta$ & 0.2 \\
Dielectric constant, $\varepsilon_{v}$ & $8.854 \times 10^{-12} \mathrm{~F} / \mathrm{m}$ \\
\hline
\end{tabular}

The variation of amplitude-frequency response of the microplate with various values of damage variable is presented in Figure 2, where DC voltage $\bar{V}_{d}$ and nondimensional material length scale parameter $l / h$ are taken as 20 and 0.2 , respectively. It should be pointed out that the current value of material length scale parameter is only a mean to illustrate microscale effect. Sophisticated experiments need to be conducted to measure material length scale parameter as it plays a significant role in microscale structures. As shown in Figure 2, the vibration amplitude becomes larger with increase of nonlinear frequency which indicates that the microplate exhibits a hardening-type behavior. It is also observed that the larger the damage variable is, the lower the nonlinear free vibration frequency becomes. Moreover, free vibration amplitude of the microplate midpoint increases as damage variable increases due to reduced stiffness of the microplate.

Influence of size effect on frequency-response curves is given in Figure 3 in which damage variable $D$ and DC voltage $\bar{V}_{d}$ are taken as 0.2 and 20, separately. It is observed that large material length scale parameter results in small amplitude and high frequency of the microplate. When especially size effect is omitted, the classical microplate model is recovered. As can be observed from Figure 3, material length scale parameter increases the difference between the results of present microplate model and the classical microplate model increases, indicating that the microscale effect induce additional rigidity for microstructure. The extra stiffness contributed by couple stress together with the classical stiffness increased the total stiffness of the microplate. Furthermore, our results are consistent with the predictions obtained by Ansari et al. [34] who studied the nonlinear vibrations properties of functionally graded Mindlin's microplates using MCST.

4.2. Forced Vibration Analysis under AC Voltage. As for dynamic analysis, (32) can be written as

$\frac{1}{4} \bar{W}_{\tau \tau}+\frac{1}{4} \bar{C} \bar{W}_{\tau}+M_{31} \bar{W}-\varphi \bar{W}^{3}=\frac{1}{4}\left(\alpha_{1}+\frac{9}{16} \alpha_{2} \bar{W}\right) \bar{V}_{a}^{2} \cos ^{2} \omega \tau$.

Similarly, the harmonic balance method is employed for solving (38) and the general solution for this equation can be given as

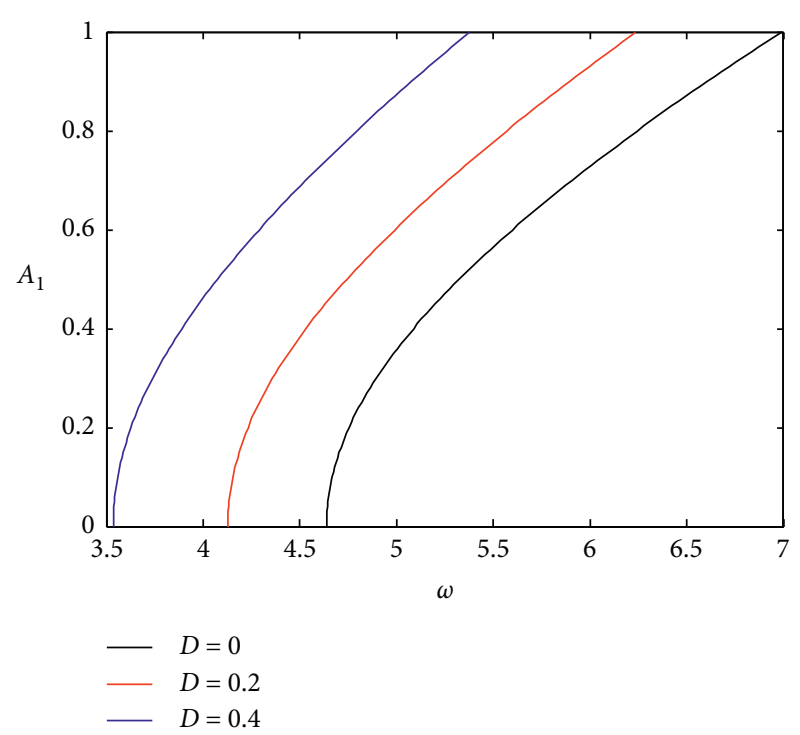

Figure 2: Damage effect on frequency-response curves of the microplate.

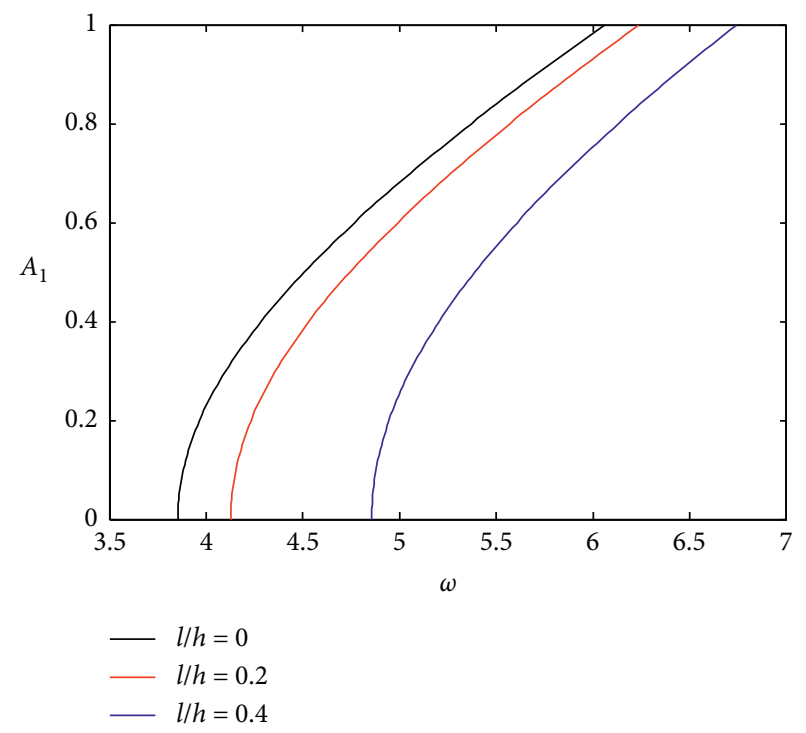

FIgURE 3: Size effect on frequency-response curves of the microplate.

$$
\begin{aligned}
W(\tau) & =A_{1} \cos \left(2 \omega \tau+\theta_{0}\right)+A_{2} \sin \left(2 \omega \tau+\theta_{0}\right) \\
& =A_{1} \cos 2 \Phi+A_{2} \sin 2 \Phi
\end{aligned}
$$

where

$$
\begin{aligned}
\cos 2 \omega \tau & =A_{3} \cos 2 \Phi+A_{4} \sin 2 \Phi, A_{3}=\cos \\
\theta_{0}, A_{4} & =\sin \theta_{0} .
\end{aligned}
$$

Substituting (39) into (38), we obtain 


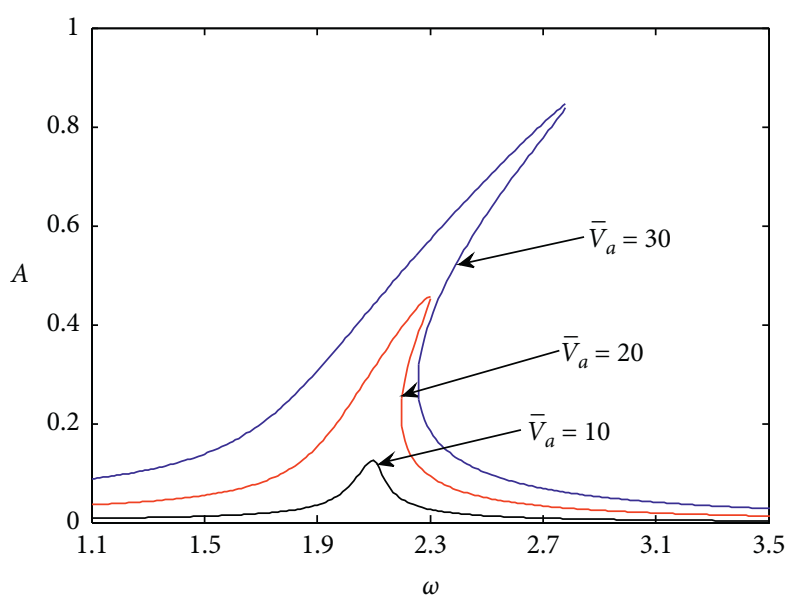

FIGURE 4: The frequency-response curves of the system for different amplitude of the AC voltage $V_{\text {AC }}$.

$$
\begin{aligned}
& \left(4 M_{31}-4 \omega^{2}-\frac{9}{32} \alpha_{2} V_{A}^{2}\right) A_{1}+2 \omega \bar{C} A_{2}-3 \varphi A_{1}\left(A_{1}^{2}+A_{2}^{2}\right)=\frac{\alpha_{1}}{2} \bar{V}_{a}^{2} A_{3} \\
& \left(4 M_{31}-4 \omega^{2}-\frac{9}{32} \alpha_{2} V_{A}^{2}\right) A_{2}-2 \omega \bar{C} A_{1}-3 \varphi A_{2}\left(A_{1}^{2}+A_{2}^{2}\right)=\frac{\alpha_{1}}{2} \bar{V}_{a}^{2} A_{4}
\end{aligned}
$$

Using $A_{1}^{2}+A_{2}^{2}=A^{2}$ and $A_{3}^{2}+A_{4}^{2}=1$, the relationship between the amplitude of the microplate midpoint and the excitation frequency can be achieved as

$$
9 \varphi^{2} A^{6}-6 \varphi\left(4 M_{31}-4 \omega^{2}-\frac{9}{32} \alpha_{2} \bar{V}_{a}^{2}\right) A^{4}+\left[\left(4 M_{31}-4 \omega^{2}-\frac{9}{32} \alpha_{2} \bar{V}_{a}^{2}\right)^{2}+4 \omega^{2} \bar{C}^{2}\right] A^{2}=\frac{\alpha_{1}^{2}}{4} \bar{V}_{a}^{4}
$$

The frequency-response curves of the system with damage variable $D$, nondimensional material length scale parameter $l / h$, and damping coefficient $\bar{C}$ are, respectively, taken as $0.2,0.2$, and 0.2 and are depicted in Figure 4 which reveals that the system still exhibits a hardening-type behavior. It is observed that the amplitude of microplate midpoint increases as excitation frequency increases from 1.1 , actually from 0 , until reaching a limit point (upper jump point), corresponding to the nonlinear resonance, and then the amplitude of microplate midpoint falls suddenly; for another, the amplitude of the microplate midpoint increases as the excitation frequency is decreased from 3.5, until reaching another limit point (lower jump point), and then the amplitude of the microplate midpoint rises abruptly. The region between the lower jump point and the upper jump point is regarded as the unstable region. This unique behavior is called as jump phenomenon which results from the nonlinearity of the dynamic system. Additionally, with the increase of the amplitude of $\mathrm{AC}$ voltage, the upper jump point tends to move towards the right side and the amplitude of the microplate midpoint increases. In other words, the nonlinear response region is enlarged by the greater external load. The jump phenomenon disappears when the amplitude of AC voltage is small, in this case, such as 10 (black line). Without enough energy input by external excitation, the dynamic system will remain stable.

Figure 5 indicates the effect of damping coefficient on the frequency-response curve of the microplate. In the numerical simulation, the nondimensional material length scale parameter $l / h$, damage variable $D$, and amplitude of AC voltage $\bar{V}_{a}$ are taken to be $0.2,0.2$, and 20 accordingly. From this figure, we can see that, by increasing the damping coefficient, the unstable region decreases, and the peak of the curves goes down. It is attributed to the fact that the energy input by external load is consumed by the dissipation system. The larger the damping coefficient is, the more energy the dynamic system expends.

Figure 6 displays damage effect on frequency-response curves of the microplate with damping coefficient $\bar{C}$, nondimensional material length scale parameter $l / h$, and amplitude of AC voltage $\bar{V}_{a}$ are set to $0.2,0.2$, and 20, respectively. It is observed that the larger the damage variable, the bigger the peak of the curve and the wider the unstable region and nonlinear response region. What is more, unlike the first two cases, the unstable region moves towards the left side when the microplate suffers more serious damage. The system does not possess enough stiffness to resist external load. The reason for this is that the stiffness of the structure is reduced by damage effect. 


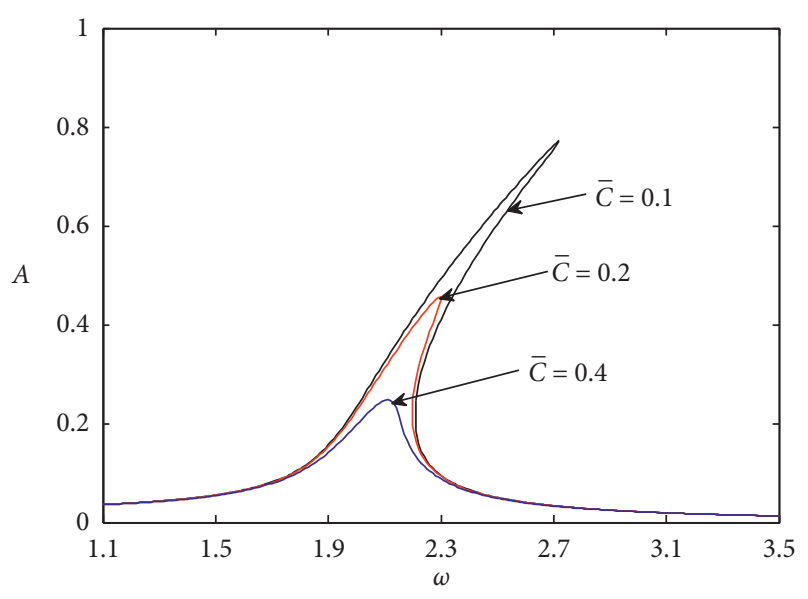

FIGURE 5: The frequency-response curves of the system for different damping coefficients $\bar{C}$.

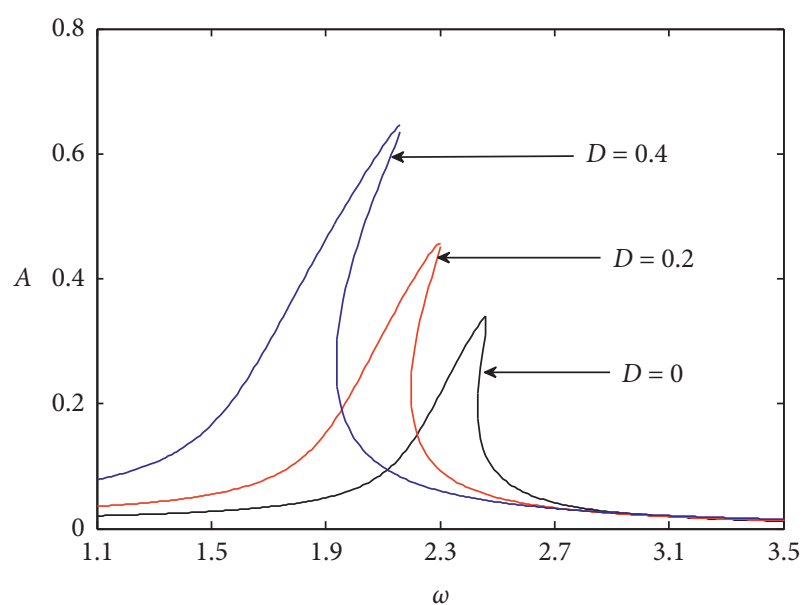

FIgURE 6: The frequency-response curves of the system for different damage variable $D$.

Figure 7 highlights the size effect on the frequencyresponse curves of dynamic system. The numerical calculations are performed by assuming $D=0.2, \bar{C}=0.2, \bar{V}_{a}=20$. It is revealed that the peak of the curve goes down slightly and the hardening influence reduces mildly when nondimensional material length scale parameter increases. In addition, the unstable region moves to the right side and diminishes as size effect becomes more obvious. Furthermore, the distinction between the results predicted by classical theory $(l / h=0)$ and nonclassical theory gets more obvious. The reason for this change was already discussed in Section 4.1. Besides, the present result is parallel to that of Ansari et al. [35], who investigated the forced vibration of functionally graded microplate based on MCST. It should be noted that horizontal axis in this work represents nondimensional external frequency, while in Ansari et al.'s work it represents the frequency ratio (the ratio of external frequency to linear first frequency).

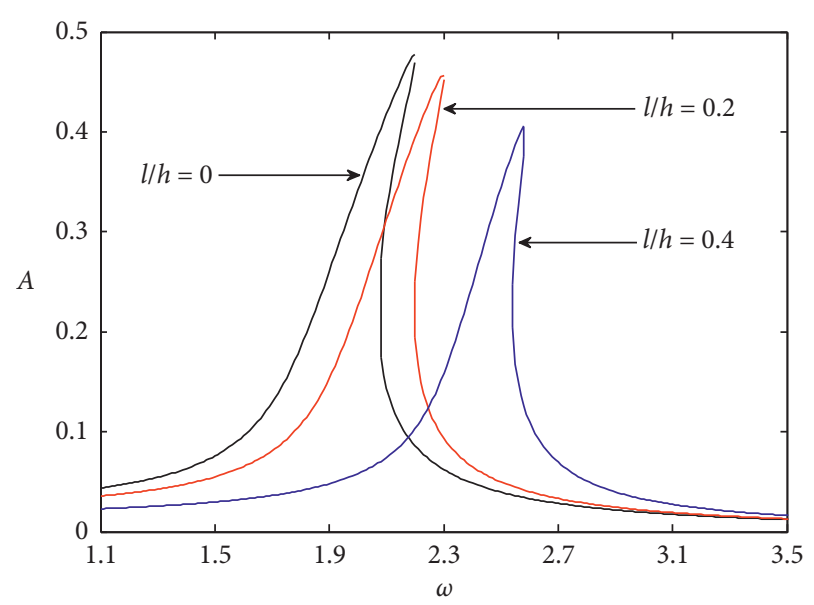

FIgURE 7: The frequency-response curves of the system for different nondimensional material length scale parameters $l / h$.

\section{Conclusions}

The nonlinear size-dependent vibration of a microplate with damage was explored by employing MCST and the strain equivalent assumption in this research. The nonlinear governing partial differential equations were transformed into nonlinear ordinary differential equations via Galerkin's scheme and further solved numerically by the harmonic balance method. Numerical results indicate that damage effect and size dependency both have obvious influences on the static and dynamic behaviors of the microplate system. It was concluded that, on one hand, the hardening-type nonlinear behavior of the microplate system enhances when it encounters damage; on the other hand, the system exhibits a weaker nonlinear behavior, greater nondimensional frequency, and lower amplitude of the microplate midpoint as size effect gets obvious.

\section{Data Availability}

The Matlab simulation and control program data used to support the findings of this study are available from the corresponding author.

\section{Conflicts of Interest}

The authors declare that there are no conflicts of interest regarding the publication of this paper.

\section{Acknowledgments}

The authors would like to acknowledge with great gratitude for the supports of the National Science Foundation of China (grant nos. 51778551 and 11272270).

\section{References}

[1] M. I. Younis and A. H. Nayfeh, "A study of the nonlinear response of a resonant micro-beam to an electric actuation," Nonlinear Dynamics, vol. 31, no. 1, pp. 91-117, 2003. 
[2] E. M. Abdel-Rahman and A. H. Nayfeh, "Secondary resonances of electrically actuated resonant microsensors," Journal of Micromechanics and Microengineering, vol. 13, no. 3, pp. 491-501, 2003.

[3] W. Zhang and G. Meng, "Nonlinear dynamical system of micro-cantilever under combined parametric and forcing excitations in MEMS," Sensors and Actuators A: Physical, vol. 119, no. 2, pp. 291-299, 2005.

[4] L. Xu and X. Jia, "Electromechanical coupled nonlinear dynamics for microbeams," Archive of Applied Mechanics, vol. 77, no. 7, pp. 485-502, 2007.

[5] W. G. Vogl and A. H. Nayfeh, "Primary resonance excitation of electrically actuated clamped circular plates," Nonlinear Dynamics, vol. 47, no. 1-3, pp. 181-192, 2006.

[6] A. H. Nayfeh, H. M. Ouakad, F. Choura, and E. M. AbdelRahman, "Nonlinear dynamics of a resonant gas sensor," Nonlinear Dynamics, vol. 59, no. 4, pp. 607-618, 2010.

[7] X. L. Jia, J. Yang, S. Kitipornchai, and C. W. Lim, "Resonance frequency response of geometrically nonlinear microswitches under electrical actuation," Journal of Sound and Vibration, vol. 331, no. 14, pp. 3397-3411, 2012.

[8] P. Kim, S. Bae, and J. Seok, "Resonant behaviors of a nonlinear cantilever beam with tip mass subject to an axial force and electrostatic excitation," International Journal of Mechanical Sciences, vol. 64, no. 1, pp. 232-257, 2012.

[9] S. Saghir and M. I. Younis, "An investigation of the static and dynamic behavior of electrically actuated rectangular microplates," International Journal of Non-linear Mechanics, vol. 85, pp. 81-93, 2016.

[10] M. Sheikhlou, R. Shabani, and G. Rezazadeh, "Nonlinear analysis of electrostatically actuated diaphragm-type micropumps," Nonlinear Dynamics, vol. 83, no. 1-2, pp. 1-11, 2016.

[11] P. M. Osterberg, Electrostatically actuated micromechanical test structure for material property measurement, $\mathrm{Ph} . \mathrm{D}$. dissertation, Massachusetts Institute of Technology, Cambridge, MA, USA, 1995.

[12] M. Arefi and A. M. Zenkour, "Size dependent electro-elastic analysis of a sandwich microbeam based on higher order sinusoidal shear deformation theory and strain gradient theory," Journal of Intelligent Material Systems and Structures, vol. 29, no. 7, pp. 27-40, 2018

[13] M. Arefi and A. M. Zenkour, "Size-dependent vibration and bending analyses of the piezomagnetic three-layer nanobeams," Applied Physics A, vol. 123, no. 3, p. 202, 2017.

[14] M. Arefi and A. M. Zenkour, "Size-dependent vibration and electro-magneto-elastic bending responses of sandwich piezomagnetic curved nanobeams," Steel and Composite Structures, vol. 29, no. 5, pp. 579-590, 2018.

[15] M. Arefi and A. M. Zenkour, "Thermo-electro-magnetomechanical bending behavior of size-dependent sandwich piezomagnetic nanoplates," Mechanics Research Communications, vol. 84, pp. 27-42, 2017.

[16] L. L. Ke, J. Yang, S. Kitipornchai, M. A. Bradford, and Y. S. Wang, "Axisymmetric nonlinear free vibration of sizedependent functionally graded annular microplates," Composites Part B: Engineering, vol. 53, no. 7, pp. 207-217, 2013.

[17] F. Yang, A. C. M. Chong, D. C. C. Lam, and P. Tong, "Couple stress based strain gradient theory for elasticity," International Journal of Solids and Structures, vol. 39, no. 10, pp. 2731-2743, 2002.

[18] M. H. Ghayesh, H. Farokhi, and M. Amabili, "Nonlinear behaviour of electrically actuated MEMS resonators,"
International Journal of Engineering Science, vol. 71, no. 10, pp. 137-155, 2013.

[19] A. G. Arani and G. S. Jafari, "Nonlinear vibration analysis of laminated composite Mindlin micro/nano-plates resting on orthotropic Pasternak medium using DQM," Applied Mathematics and Mechanics, vol. 36, no. 8, pp. 1033-1044, 2015.

[20] M. Sobhy and A. M. Zenkour, "A comprehensive study on the size-dependent hygrothermal analysis of exponentially graded microplates on elastic foundations," Mechanics of Advanced Materials and Structures, vol. 27, no. 10, pp. 816-830, 2019.

[21] M. Arefi and A. M. Zenkour, "Size-dependent free vibration and dynamic analyses of piezo-electro-magnetic sandwich nanoplates resting on viscoelastic foundation," Physica B: Condensed Matter, vol. 521, pp. 188-197, 2017.

[22] M. Arefi, M. Kiani, and A. M. Zenkour, "Size-dependent free vibration analysis of a three-layered exponentially graded nano-/micro-plate with piezomagnetic face sheets resting on Pasternak's foundation via MCST," Journal of Sandwich Structures and Materials, vol. 22, no. 1, pp. 55-86, 2020.

[23] H. Farokhi and M. H. Ghayesh, "Size-dependent behavior of electrically actuated microcantilever-based MEMS," International Journal of Mechanics \& Materials in Design, vol. 12, no. 3, pp. 1-15, 2016.

[24] M. Tahani, A. R. Askari, Y. Mohandes, and B. Hassani, "Sizedependent free vibration analysis of electrostatically pre-deformed rectangular micro-plates based on the modified couple stress theoryfied couple stress theory," International Journal of Mechanical Sciences, vol. 94-95, no. 22, pp. 185-198, 2015.

[25] A. Veysi, R. Shabani, and G. Rezazadeh, "Nonlinear vibrations of micro-doubly curved shallow shells based on the modified couple stress theoryfied couple stress theory," Nonlinear Dynamics, vol. 87, no. 3, pp. 2051-2065, 2017.

[26] B. Jalalahmadi, F. Sadeghi, and D. Peroulis, "A numerical fatigue damage model for life scatter of MEMS devices," Journal of Microelectromechanical Systems, vol. 18, no. 5, pp. 1016-1031, 2009.

[27] T. S. Slack, F. Sadeghi, and D. Peroulis, "A phenomenological discrete brittle damage-mechanics model for fatigue of MEMS devices with application to LIGA Ni," Journal of Microelectromechanical Systems, vol. 18, no. 1, pp. 119-128, 2009.

[28] A. Basu, R. P. Hennessy, G. G. Adams, and N. E. McGruer, "Hot switching damage mechanisms in MEMS contacts-evidence and understanding," Journal of Micromechanics and Microengineering, vol. 24, no. 10, p. 16, 2014.

[29] C. Chen, J. Yuan, and Y. Mao, "Post-buckling of size-dependent micro-plate considering damage effects," Nonlinear Dynamics, vol. 90, no. 2, pp. 1301-1314, 2017.

[30] J. Lemaitre, A Course on Damage Mechanics, Springer, Berlin, Germany, 1992.

[31] C. Chen, H. Hu, and L. Dai, "Nonlinear behavior and characterization of a piezoelectric laminated microbeam system," Communications in Nonlinear Science and Numerical Simulation, vol. 18, no. 5, pp. 1304-1315, 2013.

[32] A. R. Askari and M. Tahani, "Size-dependent dynamic pull-in analysis of geometric non-linear micro-plates based on the modified couple stress theory," Physica E: Low-Dimensional Systems and Nanostructures, vol. 86, pp. 262-274, 2017.

[33] A. H. Nayfeh and D. T. Mook, Nonlinear Oscillations, Springer International Publishing, New York, NY, USA, 1979. 
[34] R. Ansari, M. Faghih Shojaei, V. Mohammadi, R. Gholami, and M. A. Darabi, "Nonlinear vibrations of functionally graded Mindlin microplates based on the modified couple stress theoryfied couple stress theory," Composite Structures, vol. 114, no. 18, pp. 124-134, 2014.

[35] R. Ansari, R. Gholami, and A. Shahabodini, "Size-dependent geometrically nonlinear forced vibration analysis of functionally graded first-order shear deformable microplates," Journal of Mechanics, vol. 32, no. 5, pp. 539-554, 2016. 